chemistry laboratory can do without, and several contributions demonstrated the latest developments, which notably included Fourier transform spectroscopy-giving greatly enhanced sensitivity especially for ${ }^{13} \mathrm{C}$ and ${ }^{15} \mathrm{~N}$, and for protons in weak concentration. The fortunate owners of spectrometers based on superconducting magnets presented proton spectra with impressive resolution at frequencies greater than 200 $\mathrm{MHz}$.

Wide-line NMR was strongly represented with many contributions on topics ranging from metals and magnetic materials to ferroelectrics and polymers. Of special interest was the report by Dr M. Goldman (Saclay Laboratory) of the discovery of nuclear antiferromagnetism of the ${ }^{19} \mathrm{~F}$ nuclei in calcium fluoride when cooled to a spin temperature below $10^{-6} \mathrm{~K}$ by nuclear adiabatic demagnetization. No longer is nuclear magnetism merely a branch of paramagnetism, and no longer is the study of ordered magnetic materials the prerogative of experimenters working with materials containing unpaired electrons.

The audience had glimpses of highresolution NMR in solids too as the broadening interactions were stripped away in copper and copper compounds by high-speed magic-axis rotation (Professor E. R. Andrew, University of Nottingham), by special pulse sequences (Dr U. Haeberlen, University of Heidelberg) and by magic sandwiches (Professor J. S. Waugh, Massachusetts Institute of Technology).

A feature of the conference was the growth of the applications of magnetic resonance to systems of biological interest. Altogether there were twenty-five contributions on enzymes, membranes, cells and cell constituents, and without doubt the next international conference on magnetic resonance (in Bombay, 1974) will see further development in this important area.

\section{PROSTHESES \\ Plastics in Medicine}

from a Correspondent

THE symposium on plastics in medicine and surgery which was held at the University of Newcastle upon Tyne on September 15 and 16 was jointly organized by the north-east section of the Plastics Institute and the University of Newcastle upon Tyne Medical School with the object of bringing together the medical users of plastics and the producers.

A great deal of stress was laid on the proper selection of materials which are compatible with the internal environment of the body and the questions of toxicity, chemical stability, initiation of clot formation, allergy and carcinogenesis were discussed. It was also suggested that the physical form of the implanted material may in some circumstances be as important as its chemical structure. One conclusion was that guide lines for the testing of plastics for medical application are urgently required. There seemed some doubt, for example, as to whether testing the toxicity of a plastic to a tissue culture medium is a realistic measure of its likely performance in the body.

One session was spent discussing some of the technical problems of designing membrane oxygenations and dialysing equipment for patients with chronic renal disease. Professor R. A. M. Kenedi (University of Strathclyde), together with several other speakers, stressed the importance of mechanical design and testing of prostheses which were going to be implanted into the human body. Several surgeons recounted their clinical experiences using plastic materials for reconstructive surgery and for joint replacement.

There is no doubt that this first confrontation between members of the plastics industry and the medical profession was extremely valuable. It was clear to all the participants that, generally speaking, the plastics at present used in medicine have been drawn from the commonly available materials which were not designed with such uses in mind and that therefore much requires to be done in determining the ideal specifications that will meet the doctor's requirements. Unfortunately, just formulating a requirement is not sufficient for, as Sir Harry W. Melville, president of the Plastics Institute, said, the plastics industry thinks in terms of producing 100,000 tons of plastics at a time. Thus there is a financial difficulty because not only will a great deal of money have to be spent on development in producing special materials but also the total national demand may only be for a kilogram or two of the product.

Finally, there seemed to be general agreement that a national body should be set up to collect information from both the plastics industry and the medical profession with the object of aiding the future development of the use of plastics in medicine and surgery.

\title{
Changes in Membranes during the Cell Cycle
}

A DIVERSITY of functions is believed to be carried on at the membranes which bound all living cells and the organelles within them. Correspondingly, electron microscopy shows a variety of membrane contours, and the constituent proteins and lipids occur in all proportions. (The rolled-up membrane forming myelin is 75 per cent lipid whereas the membrane surrounding the tiny gas bubbles in some forms of alga is entirely protein.)

Another side to this diversity is the possibility that a membrane changes during the life of the cell. Evidence that the structure so varies is reported by R. E. Scott, R. C. Carter and W. R. Kidwell of the National Institutes of Health, Bethesda, in rext Wednesday's Nature New Biology. Known techniques are used to synchronize the cells in a culture so that, in step, the cells divide into two daughter cells, grow and duplicate essential molecules, and divide again. The cells are examined at various times after the initial division, and the structure of the membrane is seen to vary in a regular way.

The cells are examined by the method of freeze cleavage. A suspension of the cells in buffered glycerol-saline is frozen in liquid nitrogen and is cleaved at low temperature and under vacuum. A replica of the newly exposed surface is made by shadowing it with platinumcarbon and the replica is cleaned of the organic material and viewed in the elec- tron microscope. The question, what surface is exposed by cleaving, was previously answered by the striking micrographs of da Silva and Branton (J. Cell Biol., 45, $598 ; 1970)$ and of Tillack and Marchesi (ibid., 649). Large molecules of known appearance (ferritin and Factin) are put on the surface of red blood cells. These molecules are not seen on the cleaved surface; they appear only after ice is allowed to sublime, revealing the cell surface adjacent to the cleaved surface. Thus the surface of cleavage is within the membrane. The complementary surfaces made by the cleaving are not identical. Rather the inside half of the cell membrane shows many more particles than the extracellular half.

Scott et al. report a large variation in numbers of particles during the cycles of two kinds of cell. Shortly after division the daughter cells show the lowest number of the characteristic $85 \AA$ diameter particles per square micron of membrane. Then the number gradually doubles to the level seen before division. The numbers of particles remain in proportion on the two surfaces. Similarly there is a cyclic variation in the number of the large pores in the membrane bounding the cell nucleus. The density of pores is low shortly after division and then quickly trebles during the following growth phase. Scott et al. believe that the $85 \AA$ particles are glycoprotein and lipid. 